\title{
Change Point Detection by Sparse Parameter Estimation
}

\author{
Jiří NEUBAUER ${ }^{1}$, Vítězslav VESELÝ ${ }^{2}$ \\ ${ }^{1}$ University of Defence \\ Kounicova 65, 61200 Brno, Czech Republic \\ ${ }^{2}$ Masaryk University \\ Lipová 4la, 60200 Brno, Czech Republic \\ e-mail: jiri.neubauer@unob.cz,vesely@econ.muni.cz
}

Received: October 2009; accepted: October 2010

\begin{abstract}
The contribution is focused on change point detection in a one-dimensional stochastic process by sparse parameter estimation from an overparametrized model. A stochastic process with change in the mean is estimated using dictionary consisting of Heaviside functions. The basis pursuit algorithm is used to get sparse parameter estimates. The mentioned method of change point detection in a stochastic process is compared with several standard statistical methods by simulations.
\end{abstract}

Keywords: change point detection, overparametrized model, sparse parameter estimation.

\section{Introduction}

Chen et al. (1998) proposed a new methodology based on basis pursuit for spectral representation of signals (vectors). Instead of just representing signals as superpositions of sinusoids (the traditional Fourier representation) they suggested alternate dictionaries collections of parametrized waveforms - of which the wavelet dictionary is only the best known. A recent review paper by Bruckstein et al. (2009) demonstrates a remarkable progress in the field of sparse modeling since that time. Theoretical background for such systems (also called frames) can be found for example in Christensen (2003). In traditional Fourier expansion a presence of jumps in the signal slows down the convergence rate preventing sparsity. The Heaviside dictionary (see Chen et al., 1998) merged with the Fourier or wavelet dictionary can solve the problem quite satisfactorily. When analyzing measurements coming from real problems often some events or anomalies are manifested by abrupt changes which may not be quite apparent in their early stage, detection of hearing loss in audiological frequencies (Janušauskas et al., 2010) being an example out of many illustrating such situation.

A lot of other useful applications of sparse modeling in a variety of problems can be found in Veselý and Tonner (2006), Veselý et al. (2009) and Zelinka et al. (2004).

In Zelinka et al. (2004) kernel dictionaries are shown to be an effective alternative to traditional kernel smoothing techniques. In this paper we are using Heaviside dictionary 
in the same manner to denoise signal (the univariate time series sample path) exhibiting jumps (discontinuities) in the mean (unlike kernel smoothing where the mean is supposed to be sufficiently smooth).

Consequently, the basis pursuit approach can be proposed as an alternative to conventional statistical techniques of change point detection (see Antoch et al., 2000; Csörgö and Horváth, 1997; etc.). Unlike them, rather than on stochastic reasoning the basis pursuit relies mainly on geometric ideas where small linear correlations (normalized scalar products) of the noise vector with dictionary atoms are a dominant condition. This paper presents results of an introductory empirical comparative study for the simplest case of detecting one change point buried in additive gaussian white noise. Our primary goal is to obtain an initial judgement on the basis pursuit performance when facing up the prevailing statistical approach. In no case we aim at an exhaustive comparative analysis comprising all accompanying factors coming into play.

\section{Heaviside Dictionary for Change Point Detection}

In this section we propose the method based on basis pursuit algorithm (BPA) for the detection of the change point in the sample path $\left\{y_{t}\right\}$ in one dimensional stochastic process $\left\{Y_{t}\right\}$. We assume a deterministic functional model on a bounded interval $\mathcal{I}$ described by the dictionary $G=\left\{G_{j}\right\}_{j \in J}$ with atoms $G_{j} \in L^{2}(\mathcal{I})$ and with additive white noise $e$ on a suitable finite discrete mesh $\mathcal{T} \subset \mathcal{I}$ :

$$
Y_{t}=x_{t}+e_{t}, \quad t \in \mathcal{T}
$$

where $x \in \operatorname{sp}\left(\left\{G_{j}\right\}_{j \in J}\right),\left\{e_{t}\right\}_{t \in \mathcal{T}} \sim W N\left(0, \sigma^{2}\right), \sigma>0$, and $J$ is a big finite indexing set. Smoothed function $\hat{x}=\sum_{j \in J} \hat{\xi}_{j} G_{j}=: \mathbf{G} \hat{\xi}$ minimizes on $\mathcal{T} \ell^{1}$-penalized optimality measure $\frac{1}{2}\|\mathbf{y}-\mathbf{G} \xi\|^{2}$ as follows:

$$
\hat{\xi}=\operatorname{argmin}_{\xi \in \ell^{2}(J)} \frac{1}{2}\|\mathbf{y}-\mathbf{G} \xi\|^{2}+\lambda\|\xi\|_{1}, \quad\|\xi\|_{1}:=\sum_{j \in J}\left\|G_{j}\right\|_{2} \xi_{j},
$$

where the normalized coefficients $\left\|G_{j}\right\|_{2} \xi_{j}$ balance contribution of possibly varying atom magnitudes and $\lambda=\sigma \sqrt{2 \ln (\operatorname{card} J)}$ is a smoothing parameter chosen according to the soft-thresholding rule commonly used in wavelet theory. This choice is natural because one can prove that with any orthonormal basis $G=\left\{G_{j}\right\}_{j \in J}$ the shrinkage via softthresholding produces the same smoothing result $\hat{x}$ (see Bruckstein et al., 2009). Such approaches are also known as basis pursuit denoising (BPDN).

Solution of this minimization problem with $\lambda$ close to zero may not be sparse enough: we are searching small $F \subset J$ such that $\hat{x} \approx \sum_{j \in F} \hat{\xi}_{j} G_{j}$ is a good approximation. For that purpose we apply the following four-step procedure described in Zelinka et al. (2004) in more detail and implemented in Veselý (2001-2008). 
(A0) Choice of a raw initial estimate $\xi^{(0)}$, typically $\xi^{(0)}=\mathbf{G}^{+} \mathbf{y}$.

(A1) We improve $\xi^{(0)}$ iteratively by stopping at $\xi^{(1)}$ which satisfies optimality criterion. The solution $\xi^{(1)}$ is optimal but not sufficiently sparse in general.

(A2) Starting with $\xi^{(1)}$ we are looking for $\xi^{(2)}$ by BPDN which tends to be nearly sparse and is optimal.

(A3) We construct a sparse and optimal solution $\xi^{*}$ by removing negligible parameters and corresponding atoms from the model, namely those satisfying $\left\|G_{j}\right\|_{2}\left|\xi_{j}^{(2)}\right|<$ $\alpha\left\|\xi^{(2)}\right\|_{1}$ where $0<\alpha \ll 1$ is a suitable sparsity level, a typical choice being $\alpha=0.05$ following an analogy with the statistical significance level.

(A4) We repeat the step (A1) with the dictionary reduced according to the step (A3) and with a new initial estimate $\xi^{(0)}=\xi^{*}$. We expect to obtain a possibly improved sparse estimate $\xi^{*}$.

Hereafter we refer to this four-step algorithm as to BPA4. The steps (A1), (A2) and (A4) use Primal-Dual Barrier Method designed by Saunders (1997-2001). This up-todate sophisticated algorithm allows one to solve fairly general optimization problems minimizing convex objective subject to linear constraints. A lot of controls provide a flexible tool for adjusting the iteration process.

We build our dictionary from heaviside-shaped atoms on $L^{2}(\mathbb{R})$ derived from a fixed 'mother function' via shifting and scaling following the analogy with the construction of wavelet bases.

We construct an oversized shift-scale dictionary $G=\left\{G_{a, b}\right\}_{a \in \mathcal{A}, b \in \mathcal{B}}$ derived from the 'mother function' by varying the shift parameter $a$ and the scale (width) parameter $b$ between values from big finite sets $\mathcal{A} \subset \mathbb{R}$ and $\mathcal{B} \subset \mathbb{R}^{+}$, respectively $(J=\mathcal{A} \times \mathcal{B})$, on a bounded interval $\mathcal{I} \subset \mathbb{R}$ spanning the space $H=\operatorname{sp}\left(\left\{G_{a, b}\right\}\right)_{a \in \mathcal{A}, b \in \mathcal{B}}$, where

$$
G_{a, b}(t)=\left\{\begin{array}{lc}
1 & \text { for } t-a>b / 2 \\
2(t-a) / b & |t-a| \leqslant b / 2, b>0 \\
0 & t=a, b=0 \\
-1 & \text { otherwise. }
\end{array}\right.
$$

In the simulations below $\mathcal{I}=[0,1], \mathcal{T}=\{t / T\}$ (typically with mesh size $T=100$ ), $\mathcal{A}=\{t / T\}_{t=t_{0}}^{T-t_{0}}$ ( $t_{0}$ is a boundary trimming, $t_{0}=4$ was used in the simulations) and scale $b$ fixed to zero $(\mathcal{B}=\{0\})$. Clearly the atoms of such Heaviside dictionary are normalized on $\mathcal{I}$, i.e. $\left\|G_{a, 0}\right\|_{2}=1$. Some examples of Heaviside functions are displayed in the Fig. 1.

\section{Change Point Detection by Basis Pursuit}

We shall run BPA4 algorithm with the dictionary of Heaviside atoms. The expectations of success in recovering the true sparsest solution after step (A4) are inversely proportional 

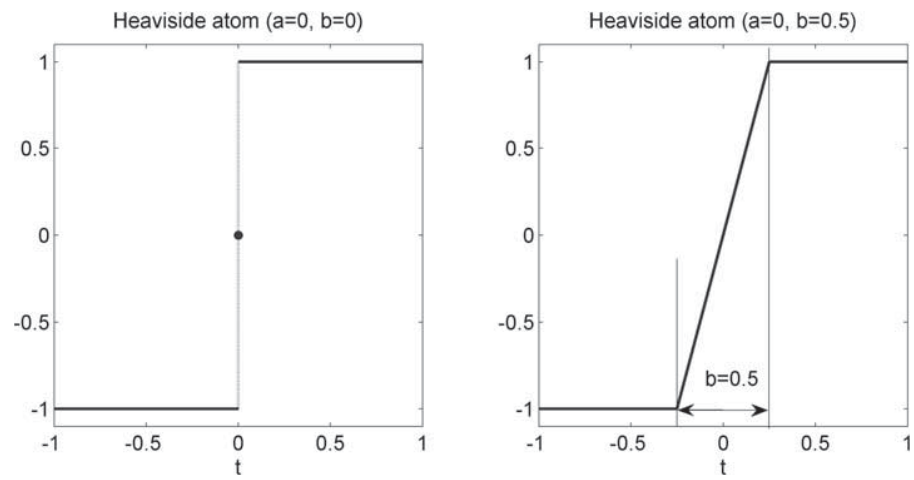

Fig. 1. Heaviside atoms with parameters $a=0, b=0$ and $a=0, b=0.5$.

to the coherence (maximal absolute correlation between distinct atoms in $G$ ) denoted as $\mu(G)$. Clearly for our dictionary this is achieved by correlation of neighbor atoms

$$
\mu(G)=\left|\int_{0}^{1} G_{a, 0}(t) G_{a+1 / T, 0}(t) d t\right|=1-\frac{1}{T}=\frac{T-1}{T}=0.99,
$$

which is very close to one and thus yields very low upper sparsity bound

$$
\frac{1}{2}\left(1+\frac{1}{\mu(G)}\right)=1+\frac{1}{2(T-1)}
$$

guaranteeing a sparsest solution with just one atom only (see Bruckstein et al., 2009, Theorems 7,8). Of course this rather conservative bound is valid for any dictionary and one can expect higher bounds with dictionaries having special structure. Let us assume that we get a sparse solution $\xi^{*}$ containing $s$ most significant atoms. To maximize the success we should assume at most one change point in $\left\{Y_{t}\right\}$ vertically centered at the origin, which guarantees existence of sparsest solution with at most one atom. With high probability this atom should belong to the family of $s$ atoms as obtained in step (A3). Among all of them we choose the atom having highest absolute correlation with the centered process $\left\{Y_{t}\right\}$.

\section{Statistical Methods of Change Point Detection}

Consider the following model

$$
Y_{t}= \begin{cases}\mu+\epsilon_{t}, & t=1,2, \ldots, c \\ \mu+\delta+\epsilon_{t}, & t=c+1, \ldots, T,\end{cases}
$$

where $\mu, \delta \neq 0, c<T$ are unknown parameters and $\epsilon_{t}$ are independent identically distributed random variables with zero mean and variance $\sigma^{2}$ and $E\left|\epsilon_{t}\right|^{2+\Delta}<\infty$ for some 
$\Delta>0$. We assume that the change point satisfies $c=[T \gamma]$ for some $\gamma \in(0,1\rangle$, where $[x]$ denotes the integer part of $x$.

Assuming $\sigma^{2}$ given, the unknown parameters $c, \mu$ and $\delta$ may be estimated by the least-squares method. The least-squares estimators $\hat{c}, \hat{\mu}$ and $\hat{\delta}$ of the parameters $c, \mu$ and $\delta$ are defined as solutions of the minimization problem

$$
\min \left\{\sum_{t=1}^{k}\left(Y_{t}-\mu\right)^{2}+\sum_{t=k+1}^{T}\left(Y_{t}-\mu-\delta\right)^{2} ; k \in\{1, \ldots, T-1\}, \mu \in \mathbb{R}, \delta \in \mathbb{R}\right\} .
$$

In other words, the unknown parameters are estimated in such a way that the sum of squares of residuals is minimal. The estimates of the parameters $\mu$ and $\delta$ are (see Antoch et al., 2000; etc.)

$$
\hat{\mu}=\bar{Y}_{\hat{c}} \quad \text { and } \quad \hat{\delta}=\bar{Y}_{\hat{c}}^{0}-\bar{Y}_{\hat{c}},
$$

where $\hat{c}$ is a solution of the maximization problem

$$
\hat{c}=\operatorname{argmax}\left\{\sqrt{\frac{T}{k(T-k)}}\left|S_{k}\right| ; k \in\{1, \ldots, T-1\}\right\},
$$

where $S_{k}=\sum_{t=1}^{k}\left(Y_{t}-\bar{Y}_{T}\right), \bar{Y}_{T}=\frac{1}{T} \sum_{t=1}^{T} Y_{t}, \bar{Y}_{\hat{c}}=\frac{1}{\hat{c}} \sum_{t=1}^{\hat{c}} Y_{t}$ and $\bar{Y}_{\hat{c}}^{0}=$ $\frac{1}{T-\hat{c}} \sum_{t=\hat{c}+1}^{T} Y_{t}$.

If we solve the given maximization problem for $[\beta T] \leqslant k \leqslant[(1-\beta) T]$, where $\beta$ is a small positive real constant less than 1 (usually $\beta \in\langle 0.01,0.1\rangle$ ), then we get the so-called trimmed estimation of the change point.

Nonparametric and robust estimates of the change point (M- and R-procedures) can be obtained by replacing the least-squares estimators and corresponding residuals by their $M$ - and $R$-counterparts.

\section{$R$-estimators of change point}

We assume the model (1) with the error terms having absolutely continuous density $f$. The rank-based estimator of $c$ is based on the partial sums $S_{k, R}=\sum_{t=1}^{k}\left(a\left(R_{t}\right)-\bar{a}_{T}\right)$, $k=1, \ldots, T$, where $R_{1}, \ldots, R_{T}$ are the ranks corresponding to $Y_{1}, \ldots, Y_{T}$, $a(1), \ldots, a(T)$ are scores and $\bar{a}_{T}=\frac{1}{T} \sum_{t=1}^{T} a(t)$. The $R$-estimator of $c$ is defined as a solution of (2), where the partial sums $S_{k}$ are substituted by the partial sums $S_{k, R}$.

\section{M-estimators of change point}

We assume the model (1) with the error terms having a common distribution $F$ that is symmetric about zero. The $M$-estimator $\hat{\mu}_{M}(\psi)$ of $\mu$ generated by score function $\psi$ is defined as a solution of the equation $\sum_{t=1}^{T} \psi\left(Y_{t}-u\right)=0$. The score function $\psi$ is assumed to be monotone and skew symmetric. The $M$-estimator $\hat{c}_{M}(\psi)$ of $c$ is defined as a solution of (2) where the partial sums $S_{k}$ are substituted by $S_{k, M}(\psi)=$ $\sum_{t=1}^{k} \psi\left(Y_{t}-\hat{\mu}_{T}(\psi)\right), k=1, \ldots, T$. 


\section{Simulation Study and Application to Real Data}

The methods mentioned above were used to compare several estimates of the change point: BP method, max-type and trimmed max-type method, $M$ - and $R$-estimators. We consider the model (1). For the purpose of simulations we put $\mu=0, \delta=1$, $T=100,200$ and 500, the error terms are independent normally distributed with zero mean and the standard deviations $\sigma=0.5,1,1.5$ and 2. We calculate simulation of this model with the change point $c=20,50$ and 80 (500 simulations for each combination of the parameters). For the purpose of comparison we standardized all simulations on the interval $[0,1]$, the change points then are $c=0.2,0.5$ and 0.8 . The results are summarized in the Tables 2-13. Each table contains descriptive statistics for $T=100$ (the first row of the part separated by a horizontal line), $T=200$ (the second row) and $T=500$ (the third row), where $\bar{x}$ denotes the sample mean, $s$ is the sample standard deviation, $x_{0.25}$ and $x_{0.75}$ are the lower and the upper sample quartiles, $x_{0.50}$ is the median, $R_{Q}$ denotes the interquartile range $x_{0.75}-x_{0.25}$. The parameter $\beta$ in the trimmed max-type method is 0.05 . The Wilcoxon scores $a(t)=t /(T+1), t=1, \ldots, T$ were used in calculation of $R$-estimators. The Huber $\psi$-function where $\psi(x)=x$ for $|x| \leqslant B$ and $\psi(x)=B \operatorname{sign}(x)$ for $|x|>B$ was chosen to get $M$-estimators of the change point $(B=20)$. For the case of white additive noise such choice seems to be sufficiently representative because additional simulations with variant choices yield comparable results. BP method was applied in two variants: BP1 with ideal centering $Y_{t}-0.5$ and BP2 with estimated centering $Y_{t}-y_{c}$, where $y_{c}=\frac{1}{2}\left(\frac{1}{m}\left(\sum_{t=1}^{m} Y_{t}+\sum_{t=T-m+1}^{100} Y_{t}\right)\right), m=15$ for $T=100, m=30$ for $T=200$ and $m=75$ for $T=500$. The parameter $\delta$ is estimated by BP1 and BP2 in the same way as statistical methods do.

Method of change point detection by BPA4 is demonstrated on simulated process (1) with the change point $c=50, \sigma=0.5, T=100$ (for BPA4 we transform it to the interval [0,1], see Fig. 2). Figure 3 shows significance plots of normalized coefficients

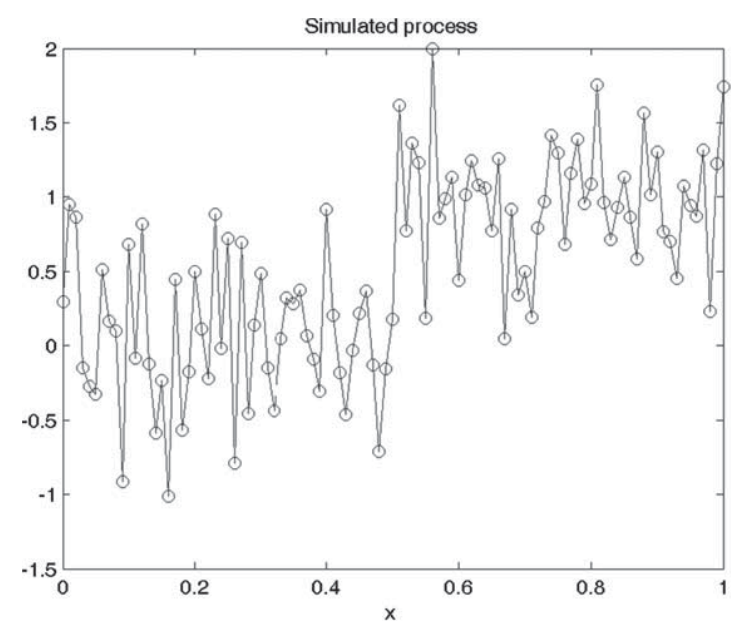

Fig. 2. Simulated process. 

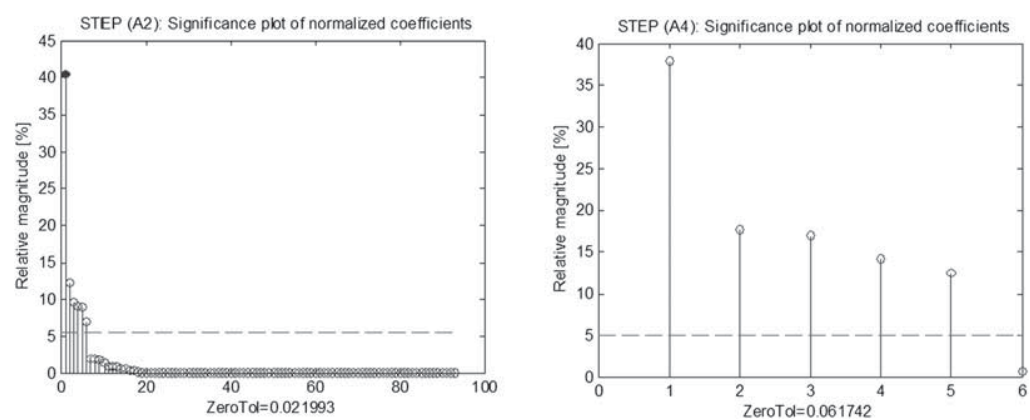

Fig. 3. Significant atoms.

Table 1

Significant atoms.

\begin{tabular}{lccl}
\hline $\begin{array}{l}\text { Atoms } \\
\text { (shift) }\end{array}$ & $\begin{array}{l}\text { Sign. coefficient } \\
\text { (above } 0.05 \text { in (A3)) }\end{array}$ & $\begin{array}{l}\text { Sign. coefficient } \\
\text { (refitted in (A4)) }\end{array}$ & $\begin{array}{l}\text { Correlation } \\
\text { (abs. value) }\end{array}$ \\
\hline 0.50 & 0.472 & 0.468 & 0.715 \\
0.66 & -0.142 & -0.219 & 0.471 \\
0.72 & 0.112 & 0.210 & 0.512 \\
0.19 & 0.104 & 0.155 & 0.373 \\
0.13 & -0.106 & -0.176 & 0.198 \\
0.73 & 0.081 & 0.008 & 0.505 \\
\hline
\end{tabular}

in decreasing order after steps (A2) and (A4) of BPA4. Dashed line denotes threshold $0.05\left\|\xi^{(2)}\right\|$ chosen in step (A3). We choose only 6 significant atoms for the simulated process, parameters and coefficient estimates of these atoms are summarized in the Table 1 . The atom with shift parameter 0.5 which corresponds to the change point $c=50$ has the highest correlation with simulated data $y_{t}$.

We use linear regression model

$$
y_{t}=b_{0}+b_{1} \cdot G_{0.5,0}(t)
$$

to get the final fit on the data set. We obtain $\hat{b}_{0}=0.511$ with the $95 \%$ confidence interval $[0.421,0.601]$ and $\hat{b}_{1}=0.463$ with the $95 \%$ confidence interval [0.373, 0.554], see Fig. 4 . Corresponding confidence intervals of regression function and confidence intervals of individual predictions are visualized by dashed lines.

We apply the methods mentioned above to the well-known dataset of the annual flows (in billions of the cubic meters) in the river Nile in Aswan (Egypt) during the years 18711970 (see Fig. 5). All estimates detect the possible change point around the year 1898. 
Table 2

Estimates of the parameters $c=0.2$ and $\delta=1$ (simulations $\sigma=0.5, \delta=1, T=100,200,500$ )

\begin{tabular}{|c|c|c|c|c|c|c|c|c|c|c|c|c|}
\hline \multirow{2}{*}{$\begin{array}{l}\sigma=0.5 \\
c=0.2\end{array}$} & \multicolumn{2}{|l|}{$\mathrm{BP} 1$} & \multicolumn{2}{|l|}{ BP2 } & \multicolumn{2}{|c|}{ Max-type } & \multicolumn{2}{|c|}{ Trimmed } & \multicolumn{2}{|c|}{$R$-estim. } & \multicolumn{2}{|c|}{$M$-estim. } \\
\hline & $\hat{c}$ & $\hat{\delta}$ & $\hat{c}$ & $\hat{\delta}$ & $\hat{c}$ & $\hat{\delta}$ & $\hat{c}$ & $\hat{\delta}$ & $\hat{c}$ & $\hat{\delta}$ & $\hat{c}$ & $\hat{\delta}$ \\
\hline \multirow[t]{3}{*}{$\bar{x}$} & 0.199 & 1.001 & 0.199 & 1.001 & 0.199 & 1.020 & 0.199 & 1.020 & 0.205 & 0.999 & 0.199 & 1.001 \\
\hline & 0.200 & 0.990 & 0.200 & 0.990 & 0.200 & 0.999 & 0.200 & 0.999 & 0.202 & 0.989 & 0.200 & 0.990 \\
\hline & 0.200 & 1.002 & 0.200 & 1.002 & 0.200 & 1.006 & 0.200 & 1.006 & 0.201 & 1.001 & 0.200 & 1.002 \\
\hline \multirow[t]{3}{*}{$s$} & 0.013 & 0.115 & 0.013 & 0.115 & 0.013 & 0.114 & 0.013 & 0.114 & 0.017 & 0.117 & 0.013 & 0.115 \\
\hline & 0.006 & 0.087 & 0.006 & 0.087 & 0.006 & 0.085 & 0.006 & 0.085 & 0.007 & 0.087 & 0.006 & 0.087 \\
\hline & 0.003 & 0.056 & 0.003 & 0.056 & 0.003 & 0.055 & 0.003 & 0.055 & 0.003 & 0.056 & 0.003 & 0.056 \\
\hline \multirow[t]{3}{*}{$x_{0.25}$} & 0.200 & 0.917 & 0.200 & 0.917 & 0.200 & 0.940 & 0.200 & 0.940 & 0.200 & 0.917 & 0.200 & 0.917 \\
\hline & 0.200 & 0.931 & 0.200 & 0.931 & 0.200 & 0.941 & 0.200 & 0.941 & 0.200 & 0.930 & 0.200 & 0.931 \\
\hline & 0.200 & 0.964 & 0.200 & 0.964 & 0.200 & 0.969 & 0.200 & 0.969 & 0.200 & 0.964 & 0.200 & 0.964 \\
\hline \multirow[t]{3}{*}{$x_{0.50}$} & 0.200 & 1.005 & 0.200 & 1.005 & 0.200 & 1.020 & 0.200 & 1.020 & 0.200 & 1.003 & 0.200 & 1.005 \\
\hline & 0.200 & 0.990 & 0.200 & 0.990 & 0.200 & 1.001 & 0.200 & 1.001 & 0.200 & 0.989 & 0.200 & 0.990 \\
\hline & 0.200 & 1.004 & 0.200 & 1.003 & 0.200 & 1.007 & 0.200 & 1.007 & 0.200 & 1.003 & 0.200 & 1.004 \\
\hline \multirow[t]{3}{*}{$x_{0.75}$} & 0.200 & 1.075 & 0.200 & 1.075 & 0.200 & 1.091 & 0.200 & 1.091 & 0.210 & 1.075 & 0.200 & 1.075 \\
\hline & 0.200 & 1.045 & 0.200 & 1.045 & 0.200 & 1.055 & 0.200 & 1.055 & 0.205 & 1.046 & 0.200 & 1.045 \\
\hline & 0.200 & 1.041 & 0.200 & 1.041 & 0.200 & 1.044 & 0.200 & 1.044 & 0.202 & 1.041 & 0.200 & 1.041 \\
\hline \multirow[t]{3}{*}{$R_{Q}$} & 0.000 & 0.157 & 0.000 & 0.158 & 0.000 & 0.151 & 0.000 & 0.151 & 0.010 & 0.158 & 0.000 & 0.157 \\
\hline & 0.000 & 0.114 & 0.000 & 0.114 & 0.000 & 0.115 & 0.000 & 0.115 & 0.005 & 0.115 & 0.000 & 0.114 \\
\hline & 0.000 & 0.077 & 0.000 & 0.077 & 0.000 & 0.075 & 0.000 & 0.075 & 0.002 & 0.078 & 0.000 & 0.077 \\
\hline
\end{tabular}

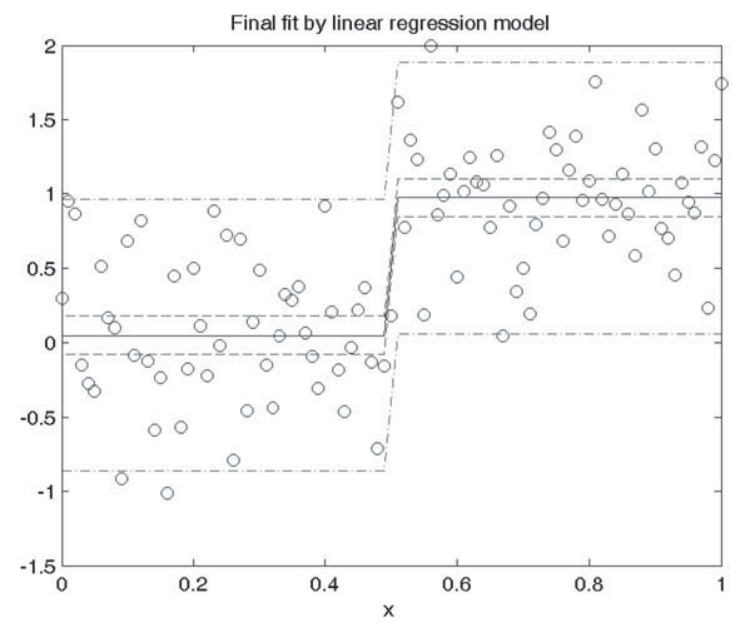

Fig. 4. Final fit by linear regression model. 
Table 3

Estimates of the parameters $c=0.5$ and $\delta=1$ (simulations $\sigma=0.5, T=100,200,500$ )

\begin{tabular}{|c|c|c|c|c|c|c|c|c|c|c|c|c|}
\hline \multirow{2}{*}{$\begin{array}{l}\sigma=0.5 \\
c=0.5\end{array}$} & \multicolumn{2}{|l|}{ BP1 } & \multicolumn{2}{|l|}{$\mathrm{BP} 2$} & \multicolumn{2}{|c|}{ Max-type } & \multicolumn{2}{|c|}{ Trimmed } & \multicolumn{2}{|c|}{$R$-estim. } & \multicolumn{2}{|c|}{$M$-estim. } \\
\hline & $\hat{c}$ & $\hat{\delta}$ & $\bar{c}$ & $\hat{\delta}$ & $\hat{c}$ & $\hat{\delta}$ & $\hat{c}$ & $\hat{\delta}$ & $\overline{\hat{c}}$ & $\hat{\delta}$ & $\overline{\hat{c}}$ & $\hat{\delta}$ \\
\hline \multirow[t]{3}{*}{$\bar{x}$} & 0.499 & 0.992 & 0.499 & 0.992 & 0.500 & 1.015 & 0.500 & 1.015 & 0.499 & 0.992 & 0.500 & 0.992 \\
\hline & 0.500 & 0.987 & 0.500 & 0.987 & 0.500 & 0.999 & 0.500 & 0.999 & 0.500 & 0.987 & 0.500 & 0.987 \\
\hline & 0.500 & 0.996 & 0.500 & 0.996 & 0.500 & 1.001 & 0.500 & 1.001 & 0.500 & 0.996 & 0.500 & 0.996 \\
\hline \multirow[t]{3}{*}{$s$} & 0.012 & 0.099 & 0.012 & 0.099 & 0.012 & 0.099 & 0.012 & 0.099 & 0.014 & 0.099 & 0.012 & 0.099 \\
\hline & 0.007 & 0.068 & 0.007 & 0.068 & 0.007 & 0.068 & 0.007 & 0.068 & 0.007 & 0.068 & 0.007 & 0.068 \\
\hline & 0.003 & 0.045 & 0.003 & 0.045 & 0.003 & 0.045 & 0.003 & 0.045 & 0.003 & 0.045 & 0.003 & 0.045 \\
\hline \multirow[t]{3}{*}{$x_{0.25}$} & 0.500 & 0.930 & 0.500 & 0.930 & 0.500 & 0.952 & 0.500 & 0.952 & 0.500 & 0.930 & 0.500 & 0.930 \\
\hline & 0.500 & 0.937 & 0.500 & 0.937 & 0.500 & 0.950 & 0.500 & 0.950 & 0.500 & 0.938 & 0.500 & 0.937 \\
\hline & 0.500 & 0.966 & 0.500 & 0.966 & 0.500 & 0.970 & 0.500 & 0.970 & 0.500 & 0.966 & 0.500 & 0.966 \\
\hline \multirow[t]{3}{*}{$x_{0.50}$} & 0.500 & 0.994 & 0.500 & 0.994 & 0.500 & 1.018 & 0.500 & 1.018 & 0.500 & 0.994 & 0.500 & 0.994 \\
\hline & 0.500 & 0.987 & 0.500 & 0.987 & 0.500 & 1.000 & 0.500 & 1.000 & 0.500 & 0.987 & 0.500 & 0.987 \\
\hline & 0.500 & 0.996 & 0.500 & 0.996 & 0.500 & 1.001 & 0.500 & 1.001 & 0.500 & 0.996 & 0.500 & 0.996 \\
\hline \multirow[t]{3}{*}{$x_{0.75}$} & 0.500 & 1.055 & 0.500 & 1.055 & 0.500 & 1.076 & 0.500 & 1.076 & 0.500 & 1.054 & 0.500 & 1.055 \\
\hline & 0.500 & 1.030 & 0.500 & 1.030 & 0.500 & 1.044 & 0.500 & 1.044 & 0.500 & 1.031 & 0.500 & 1.030 \\
\hline & 0.500 & 1.028 & 0.500 & 1.028 & 0.500 & 1.032 & 0.500 & 1.032 & 0.500 & 1.028 & 0.500 & 1.028 \\
\hline \multirow[t]{3}{*}{$R_{Q}$} & 0.000 & 0.125 & 0.000 & 0.125 & 0.000 & 0.124 & 0.000 & 0.124 & 0.000 & 0.124 & 0.000 & 0.125 \\
\hline & 0.000 & 0.093 & 0.000 & 0.0 & 0.000 & 0.094 & 0.000 & 0.094 & 0.000 & 0.093 & 0.000 & 0.093 \\
\hline & 0.000 & 0.062 & 0.000 & 0.062 & 0.000 & 0.062 & 0.000 & 0.062 & 0.000 & 0.062 & 0.000 & 0.062 \\
\hline
\end{tabular}

Table 4

Estimates of the parameters $c=0.8$ and $\delta=1$ (simulations $\sigma=0.5, T=100,200,500$ )

\begin{tabular}{|c|c|c|c|c|c|c|c|c|c|c|c|c|}
\hline \multirow{2}{*}{$\begin{array}{l}\sigma=0.5 \\
c=0.8\end{array}$} & \multicolumn{2}{|l|}{ BP1 } & \multicolumn{2}{|l|}{ BP2 } & \multicolumn{2}{|c|}{ Max-type } & \multicolumn{2}{|c|}{ Trimmed } & \multicolumn{2}{|c|}{$R$-estim. } & \multicolumn{2}{|c|}{$M$-estim. } \\
\hline & $\hat{c}$ & $\hat{\delta}$ & $\hat{c}$ & $\hat{\delta}$ & $\bar{c}$ & $\hat{\delta}$ & $\overline{\hat{c}}$ & $\hat{\delta}$ & $\hat{c}$ & $\hat{\delta}$ & $\overline{\hat{c}}$ & $\hat{\delta}$ \\
\hline \multirow[t]{3}{*}{$\bar{x}$} & 0.799 & 0.966 & 0.799 & 0.966 & 0.799 & 1.021 & 0.799 & 1.021 & 0.794 & 0.953 & 0.799 & 0.967 \\
\hline & 0.800 & 0.973 & 0.800 & 0.973 & 0.799 & 1.001 & 0.799 & 1.001 & 0.798 & 0.967 & 0.799 & 0.973 \\
\hline & 0.800 & 0.991 & 0.800 & 0.991 & 0.800 & 1.002 & 0.800 & 1.002 & 0.799 & 0.988 & 0.800 & 0.991 \\
\hline \multirow[t]{3}{*}{$s$} & 0.016 & 0.123 & 0.016 & 0.123 & 0.016 & 0.125 & 0.016 & 0.125 & 0.020 & 0.121 & 0.016 & 0.123 \\
\hline & 0.006 & 0.082 & 0.006 & 0.082 & 0.006 & 0.083 & 0.006 & 0.083 & 0.007 & 0.082 & 0.006 & 0.082 \\
\hline & 0.003 & 0.057 & 0.003 & 0.057 & 0.003 & 0.057 & 0.003 & 0.057 & 0.003 & 0.057 & 0.003 & 0.057 \\
\hline \multirow[t]{3}{*}{$x_{0.25}$} & 0.800 & 0.891 & 0.800 & 0.891 & 0.800 & 0.944 & 0.800 & 0.944 & 0.790 & 0.879 & 0.800 & 0.891 \\
\hline & 0.800 & 0.91 & 0.800 & 0.914 & 0.800 & 0.944 & 0.800 & 0.944 & 0.795 & 0.906 & 0.800 & 0.914 \\
\hline & 0.800 & 0.951 & 0.800 & 0.951 & 0.800 & 0.964 & 0.800 & 0.964 & 0.798 & 0.948 & 0.800 & 0.951 \\
\hline \multirow[t]{3}{*}{$x_{0.50}$} & 0.800 & 0.972 & 0.800 & 0.972 & 0.800 & 1.026 & 0.800 & 1.026 & 0.800 & 0.954 & 0.800 & 0.972 \\
\hline & 0.800 & 0.97 & 0 & 0. & 0.800 & 1.0 & 0.800 & 1.0 & 0.800 & 0.9 & 0.800 & 0.974 \\
\hline & 0.800 & 0.990 & 0.800 & 0.990 & 0.800 & 1.002 & 0.800 & 1.002 & 0.800 & 0.986 & 0.800 & 0.990 \\
\hline \multirow[t]{3}{*}{$x_{0.75}$} & 0.800 & 1.046 & 0.800 & 1.046 & 0.800 & 1.103 & 0.800 & 1.103 & 0.800 & 1.035 & 0.800 & 1.046 \\
\hline & 0.800 & 1.028 & 0.800 & 1.028 & 0.800 & 1.059 & 0.800 & 1.059 & 0.800 & 1.021 & 0.800 & 1.028 \\
\hline & 0.800 & 1.029 & 0.800 & 1.029 & 0.800 & 1.039 & 0.800 & 1.039 & 0.800 & 1.024 & 0.800 & 1.029 \\
\hline \multirow[t]{3}{*}{$R_{Q}$} & 0.000 & 0.156 & 0.000 & 0.155 & 0.000 & 0.159 & 0.000 & 0.159 & 0.010 & 0.156 & 0.000 & 0.155 \\
\hline & 0.000 & & & & 0.000 & 0.116 & 0.000 & 0.116 & 0.005 & & 0.000 & 0.114 \\
\hline & 0.000 & 0.078 & 0.000 & 0.078 & 0.000 & 0.075 & 0.000 & 0.075 & 0.002 & 0.076 & 0.000 & 0.078 \\
\hline
\end{tabular}


Table 5

Estimates of the parameters $c=0.2$ and $\delta=1$ (simulations $\sigma=1, T=100,200,500$ )

\begin{tabular}{|c|c|c|c|c|c|c|c|c|c|c|c|c|}
\hline \multirow{2}{*}{$\begin{array}{l}\sigma=1 \\
c=0.2\end{array}$} & \multicolumn{2}{|l|}{ BP1 } & \multicolumn{2}{|l|}{ BP2 } & \multicolumn{2}{|c|}{ Max-type } & \multicolumn{2}{|c|}{ Trimmed } & \multicolumn{2}{|c|}{$R$-estim. } & \multicolumn{2}{|c|}{$M$-estim. } \\
\hline & $\overline{\hat{c}}$ & $\hat{\delta}$ & $\hat{c}$ & $\hat{\delta}$ & $\hat{c}$ & $\hat{\delta}$ & $\hat{c}$ & $\widehat{\delta}$ & $\hat{c}$ & $\hat{\delta}$ & $\hat{c}$ & $\widehat{\hat{\delta}}$ \\
\hline \multirow[t]{3}{*}{$\bar{x}$} & 0.216 & 1.027 & 0.220 & 1.024 & 0.217 & 1.078 & 0.218 & 1.075 & 0.228 & 1.021 & 0.217 & 1.030 \\
\hline & 0.201 & 1.015 & 0.201 & 1.015 & 0.201 & 1.037 & 0.201 & 1.037 & 0.204 & 1.013 & 0.201 & 1.015 \\
\hline & 0.201 & 1.002 & 0.202 & 1.000 & 0.200 & 1.012 & 0.200 & 1.012 & 0.202 & 1.001 & 0.200 & 1.002 \\
\hline \multirow[t]{3}{*}{$s$} & 0.091 & 0.253 & 0.101 & 0.259 & 0.098 & 0.296 & 0.096 & 0.248 & 0.114 & 0.284 & 0.098 & 0.274 \\
\hline & 0.028 & 0.179 & 0.028 & 0.179 & 0.028 & 0.177 & 0.028 & 0.177 & 0.033 & 0.179 & 0.028 & 0.179 \\
\hline & 0.012 & 0.113 & 0.016 & 0.116 & 0.011 & 0.113 & 0.011 & 0.113 & 0.014 & 0.114 & 0.011 & 0.113 \\
\hline \multirow[t]{3}{*}{$x_{0.25}$} & 0.190 & 0.873 & 0.180 & 0.873 & 0.190 & 0.920 & 0.190 & 0.919 & 0.190 & 0.875 & 0.190 & 0.874 \\
\hline & 0.195 & 0.886 & 0.195 & 0.887 & 0.195 & 0.906 & 0.195 & 0.906 & 0.195 & 0.88 & 0.195 & 0.886 \\
\hline & 0.198 & 0.923 & 0.196 & 0.922 & 0.198 & 0.930 & 0.198 & 0.930 & 0.198 & 0.923 & 0.198 & 0.923 \\
\hline \multirow[t]{3}{*}{$x_{0.50}$} & 0.200 & 1.023 & 0.200 & 1.020 & 0.200 & 1.065 & 0.200 & 1.063 & 0.200 & 1.019 & 0.200 & 1.024 \\
\hline & 0.200 & 1.026 & 0.200 & 1.024 & 0.200 & 1.051 & 0.200 & 1.051 & 0.200 & 1.023 & 0.200 & 1.026 \\
\hline & 0.200 & 1.007 & 0.200 & 1.002 & 0.200 & 1.015 & 0.200 & 1.015 & 0.200 & 1.006 & 0.200 & 1.008 \\
\hline \multirow[t]{3}{*}{$x_{0.75}$} & 0.220 & 1.200 & 0.220 & 1.198 & 0.220 & 1.255 & 0.220 & 1.248 & 0.230 & 1.194 & 0.220 & 1.201 \\
\hline & 0.205 & 1.144 & 0.205 & 1.144 & 0.205 & 1.166 & 0.205 & 1.166 & 0.210 & 1.144 & 0.205 & 1.145 \\
\hline & 0.202 & 1.083 & 0.204 & 1.084 & 0.202 & 1.089 & 0.202 & 1.089 & 0.204 & 1.081 & 0.202 & 1.083 \\
\hline \multirow[t]{3}{*}{$R_{Q}$} & 0.030 & 0.327 & 0.040 & 0.326 & 0.030 & 0.334 & 0.030 & 0.330 & 0.040 & 0.319 & 0.030 & 0.327 \\
\hline & 0.010 & 0.259 & 0.010 & & 0.010 & 0.260 & 0.010 & 0.260 & 0.015 & 0.256 & 0.010 & 0.259 \\
\hline & 0.004 & 0.160 & 0.008 & 0.162 & 0.004 & 0.159 & 0.004 & 0.159 & 0.006 & 0.158 & 0.004 & 0.160 \\
\hline
\end{tabular}

Table 6

Estimates of the parameters $c=0.5$ and $\delta=1$ (simulations $\sigma=1, T=100,200,500$ )

\begin{tabular}{|c|c|c|c|c|c|c|c|c|c|c|c|c|}
\hline \multirow{2}{*}{$\begin{array}{l}\sigma=1 \\
c=0.5\end{array}$} & \multicolumn{2}{|l|}{ BP1 } & \multicolumn{2}{|l|}{$\mathrm{BP} 2$} & \multicolumn{2}{|c|}{ Max-type } & \multicolumn{2}{|c|}{ Trimmed } & \multicolumn{2}{|c|}{$R$-estim. } & \multicolumn{2}{|c|}{$M$-estim. } \\
\hline & $\hat{c}$ & $\hat{\delta}$ & $\hat{c}$ & $\hat{\delta}$ & $\hat{c}$ & $\hat{\delta}$ & $\hat{c}$ & $\hat{\delta}$ & $\hat{c}$ & $\hat{\hat{\delta}}$ & $\hat{c}$ & $\hat{\hat{\delta}}$ \\
\hline \multirow[t]{3}{*}{$\bar{x}$} & 0.499 & 1.016 & 0.498 & 1.015 & 0.499 & 1.058 & 0.499 & 1.058 & 0.498 & 1.017 & 0.499 & 1.018 \\
\hline & 0.502 & 0.986 & 0.502 & 0.986 & 0.503 & 1.008 & 0.503 & 1.008 & 0.502 & 0.985 & 0.503 & 0.986 \\
\hline & 0.500 & 0.994 & 0.500 & 0.994 & 0.500 & 1.003 & 0.500 & 1.003 & 0.500 & 0.994 & 0.500 & 0.994 \\
\hline \multirow[t]{3}{*}{$s$} & 0.073 & 0.204 & 0.074 & 0.204 & 0.070 & 0.201 & 0.070 & 0.201 & 0.075 & 0.200 & 0.070 & 0.203 \\
\hline & 0.036 & 0.132 & 0.036 & 0.1 & 0.035 & 0.131 & 0.035 & 0.131 & 0.031 & 0.132 & 0.035 & 0.132 \\
\hline & 0.014 & 0.088 & 0.014 & 0.088 & 0.010 & 0.087 & 0.010 & 0.087 & 0.011 & 0.087 & 0.010 & 0.087 \\
\hline \multirow[t]{3}{*}{$x_{0.25}$} & 0.480 & 0.875 & 0.480 & 0.875 & 0.480 & 0.915 & 0.480 & 0.915 & 0.480 & 0.877 & 0.480 & 0.876 \\
\hline & 0.495 & 0.90 & 0.495 & 0.902 & 0.495 & 0.918 & 0.495 & 0.9 & 0.495 & 0.902 & 0.495 & 0.902 \\
\hline & 0.498 & 0.933 & 0.498 & 0.933 & 0.498 & 0.943 & 0.498 & 0.943 & 0.496 & 0.934 & 0.498 & 0.934 \\
\hline \multirow[t]{3}{*}{$x_{0.50}$} & 0.500 & 1.012 & 0.500 & 1.011 & 0.500 & 1.053 & 0.500 & 1.053 & 0.500 & 1.010 & 0.500 & 1.011 \\
\hline & 0.500 & 0.981 & 0.500 & 0.981 & 0.500 & 0.998 & 0.500 & 0.998 & 0.500 & 0.980 & 0.500 & 0.981 \\
\hline & 0.500 & 0.997 & 0.500 & 0.997 & 0.500 & 1.008 & 0.500 & 1.008 & 0.500 & 0.997 & 0.500 & 0.997 \\
\hline \multirow[t]{3}{*}{$x_{0.75}$} & 0.510 & 1.148 & 0.510 & 1.148 & 0.510 & 1.182 & 0.510 & 1.182 & 0.515 & 1.146 & 0.510 & 1.149 \\
\hline & 0.510 & 1.07 & 0.510 & 1.075 & 0.510 & 1.096 & 0.510 & 1.096 & 0.510 & 1.074 & 0.510 & 1.076 \\
\hline & 0.504 & 1.054 & 0.504 & 1.054 & 0.502 & 1.065 & 0.502 & 1.065 & 0.502 & 1.053 & 0.502 & 1.054 \\
\hline \multirow[t]{3}{*}{$R_{Q}$} & 0.030 & 0.274 & 0.030 & 0.274 & 0.030 & 0.267 & 0.030 & 0.267 & 0.035 & 0.269 & 0.030 & 0.273 \\
\hline & 0.015 & & & 0.173 & 0.015 & 0.178 & 0.015 & & 0.015 & 0.173 & 0.015 & 0.174 \\
\hline & 0.006 & 0.121 & 0.006 & 0.121 & 0.004 & 0.122 & 0.004 & 0.122 & 0.006 & 0.119 & 0.004 & 0.120 \\
\hline
\end{tabular}


Table 7

Estimates of the parameters $c=0.8$ and $\delta=1$ (simulations $\sigma=1, T=100,200,500$ )

\begin{tabular}{|c|c|c|c|c|c|c|c|c|c|c|c|c|}
\hline \multirow{2}{*}{$\begin{array}{l}\sigma=1 \\
c=0.8\end{array}$} & \multicolumn{2}{|l|}{ BP1 } & \multicolumn{2}{|l|}{ BP2 } & \multicolumn{2}{|c|}{ Max-type } & \multicolumn{2}{|c|}{ Trimmed } & \multicolumn{2}{|c|}{$R$-estim. } & \multicolumn{2}{|c|}{$M$-estim. } \\
\hline & $\hat{\hat{c}}$ & $\hat{\delta}$ & $\overline{\hat{c}}$ & $\hat{\delta}$ & $\hat{c}$ & $\hat{\delta}$ & $\hat{c}$ & $\hat{\delta}$ & $\overline{\hat{c}}$ & $\hat{\delta}$ & $\hat{c}$ & $\hat{\delta}$ \\
\hline \multirow[t]{3}{*}{$\bar{x}$} & 0.784 & 0.986 & 0.783 & 0.992 & 0.786 & 1.078 & 0.785 & 1.070 & 0.777 & 0.971 & 0.786 & 0.998 \\
\hline & 0.795 & 0.981 & 0.795 & 0.981 & 0.796 & 1.026 & 0.795 & 1.021 & 0.792 & 0.974 & 0.796 & 0.983 \\
\hline & 0.799 & 0.990 & 0.799 & 0.991 & 0.799 & 1.008 & 0.799 & 1.008 & 0.798 & 0.988 & 0.799 & 0.991 \\
\hline \multirow[t]{3}{*}{$s$} & 0.084 & 0 . & 1 & 0 & 89 & 0 & 81 & 58 & 95 & 19 & 0.089 & 0.258 \\
\hline & 0.053 & 0.179 & 0.053 & 0.179 & 0.054 & 0.200 & 0.053 & 0.181 & 0.061 & 0.176 & 0.054 & 0.179 \\
\hline & 0.013 & 0.108 & 0.014 & 0.109 & 0.011 & 0.108 & 0.011 & 0.108 & 0.012 & 0.106 & 0.011 & 0.107 \\
\hline \multirow[t]{3}{*}{$x_{0.25}$} & 0.780 & 0.805 & 0.780 & 0.814 & 0.780 & 0.889 & 0.780 & 0.881 & 0.780 & 0.799 & 0.780 & 0.818 \\
\hline & 0.790 & 0.859 & 0.790 & 0.859 & 0.790 & 0.902 & 0.790 & 0.902 & 0.790 & 0.858 & 0.790 & 0.862 \\
\hline & 0.796 & 0.918 & 0.796 & 0.918 & 0.796 & 0.933 & 0.796 & 0.933 & 0.796 & 0.916 & 0.796 & 0.916 \\
\hline \multirow[t]{3}{*}{$x_{0.50}$} & 0.800 & 0.989 & 0.800 & 0.997 & 0.800 & 1.075 & 0.800 & 1.071 & 0.800 & 0.972 & 0.800 & 1.000 \\
\hline & 0.800 & 0.996 & 0.800 & 0.996 & 0.800 & 1.035 & 0.800 & 1.033 & 0.800 & 0.985 & 0.800 & 0.995 \\
\hline & 0.800 & 0.987 & 0.800 & 0.987 & 0.800 & 1.002 & 0.800 & 1.002 & 0.800 & 0.983 & 0.800 & 0.985 \\
\hline \multirow[t]{3}{*}{$x_{0.75}$} & 0.810 & 1.147 & 0.820 & 1.155 & 0.815 & 1.229 & 0.810 & 1.227 & 0.810 & 1.123 & 0.815 & 1.159 \\
\hline & 0.810 & 1.104 & 0.810 & 1.104 & 0.810 & 1.145 & 0.810 & 1.145 & 0.805 & 1.095 & 0.810 & 1.105 \\
\hline & 0.802 & 1.052 & 0.802 & 1.054 & 0.802 & 1.072 & 0.802 & 1.072 & 0.802 & 1.050 & 0.802 & 1.059 \\
\hline \multirow[t]{3}{*}{$R_{Q}$} & 0.030 & 0.342 & 0.040 & 0.341 & 0.035 & 0.341 & 0.030 & 0.346 & 0.030 & 0.324 & 0.035 & 0.340 \\
\hline & 0.020 & 0.245 & 0.020 & 0.245 & 0.020 & 0.243 & 0.020 & & 0.015 & 0.237 & 0.020 & 0.243 \\
\hline & 0.006 & 0.134 & 0.006 & 0.136 & 0.006 & 0.139 & 0.006 & 0.139 & 0.006 & 0.134 & 0.006 & 0.143 \\
\hline
\end{tabular}

Table 8

Estimates of the parameters $c=0.2$ and $\delta=1$ (simulations $\sigma=1.5, T=100,200,500$ )

\begin{tabular}{|c|c|c|c|c|c|c|c|c|c|c|c|c|}
\hline \multirow{2}{*}{$\begin{array}{l}\sigma=1.5 \\
c=0.2\end{array}$} & \multicolumn{2}{|l|}{ BP1 } & \multicolumn{2}{|l|}{$\mathrm{BP} 2$} & \multicolumn{2}{|c|}{ Max-type } & \multicolumn{2}{|c|}{ Trimmed } & \multicolumn{2}{|c|}{$R$-estim. } & \multicolumn{2}{|c|}{$M$-estim. } \\
\hline & $\hat{c}$ & $\hat{\delta}$ & $\hat{c}$ & $\widehat{\hat{\delta}}$ & $\hat{c}$ & $\hat{\delta}$ & $\hat{c}$ & $\hat{\delta}$ & $\hat{c}$ & $\hat{\delta}$ & $\hat{c}$ & $\hat{\delta}$ \\
\hline \multirow[t]{3}{*}{$\bar{x}$} & 0.266 & 1.087 & 0.270 & 1.074 & 0.272 & 1.245 & 0.265 & 1.166 & 0.283 & 1.113 & 0.272 & 1.145 \\
\hline & 0.225 & 1.045 & 0.224 & 1.045 & 0.227 & 1.081 & 0.225 & 1.084 & 0.227 & 1.046 & 0.227 & 1.047 \\
\hline & 0.202 & 1.028 & 0.204 & 1.021 & 0.203 & 1.045 & 0.202 & 1.044 & 0.204 & 1.024 & 0.203 & 1.030 \\
\hline \multirow[t]{3}{*}{$s$} & 0.182 & 0.471 & 0.186 & 0.482 & 0.208 & 0.711 & 0.185 & 0.514 & 0.211 & 0.547 & 0.208 & 0.598 \\
\hline & 0.116 & 0.282 & 16 & 0.284 & 0.135 & 0.526 & 0.117 & 0.282 & 0.117 & 0.332 & 0.135 & 0.407 \\
\hline & 0.032 & 0.180 & 0.040 & 0.190 & 0.046 & 0.178 & 0.031 & 0.179 & 0.031 & 0.176 & 0.046 & 0.178 \\
\hline \multirow[t]{3}{*}{$x_{0.25}$} & 0.180 & 0.859 & 0.180 & 0.836 & 0.170 & 0.965 & 0.180 & 0.936 & 0.180 & 0.880 & 0.170 & 0.880 \\
\hline & 0.185 & 0.87 & 185 & 0.87 & 0.185 & 0.92 & 0.185 & 0.91 & 0.185 & 0.87 & 0.185 & 0.884 \\
\hline & 0.194 & 0.914 & 0.192 & 0.904 & 0.192 & 0.928 & 0.192 & 0.928 & 0.194 & 0.911 & 0.192 & 0.915 \\
\hline \multirow[t]{3}{*}{$x_{0.50}$} & 0.210 & 1.088 & 0.210 & 1.084 & 0.210 & 1.217 & 0.210 & 1.173 & 0.210 & 1.103 & 0.210 & 1.123 \\
\hline & 0.200 & 1.042 & 0.200 & 1.043 & 0.200 & 1.086 & 0.200 & 1.081 & 0.205 & 1.042 & 0.200 & 1.048 \\
\hline & 0.200 & 1.027 & 0.200 & 1.025 & 0.200 & 1.041 & 0.200 & 1.040 & 0.200 & 1.027 & 0.200 & 1.028 \\
\hline \multirow[t]{3}{*}{$x_{0.75}$} & 0.280 & 1.346 & 0.290 & 1.333 & 0.280 & 1.488 & 0.280 & 1.447 & 0.295 & 1.374 & 0.280 & 1.400 \\
\hline & 0.225 & 1.204 & 0.225 & 1.196 & 0.225 & 1.268 & 0.225 & 1.253 & 0.228 & 1.196 & 0.225 & 1.219 \\
\hline & 0.208 & 1.145 & 0.210 & 1.143 & 0.208 & 1.163 & 0.208 & 1.163 & 0.210 & 1.142 & 0.208 & 1.145 \\
\hline \multirow[t]{3}{*}{$R_{Q}$} & 0.100 & & & & & & 0.100 & & 0.115 & & 0.110 & 0.520 \\
\hline & 0.040 & 0.3 & 0.040 & & 0.040 & & 0.040 & & 0.043 & 0.324 & 0.040 & 0.335 \\
\hline & 0.014 & 0.231 & 0.018 & 0.239 & 0.016 & 0.235 & 0.016 & 0.235 & 0.016 & 0.231 & 0.016 & 0.230 \\
\hline
\end{tabular}


Table 9

Estimates of the parameters $c=0.5$ and $\delta=1$ (simulations $\sigma=1.5, T=100,200,500$ )

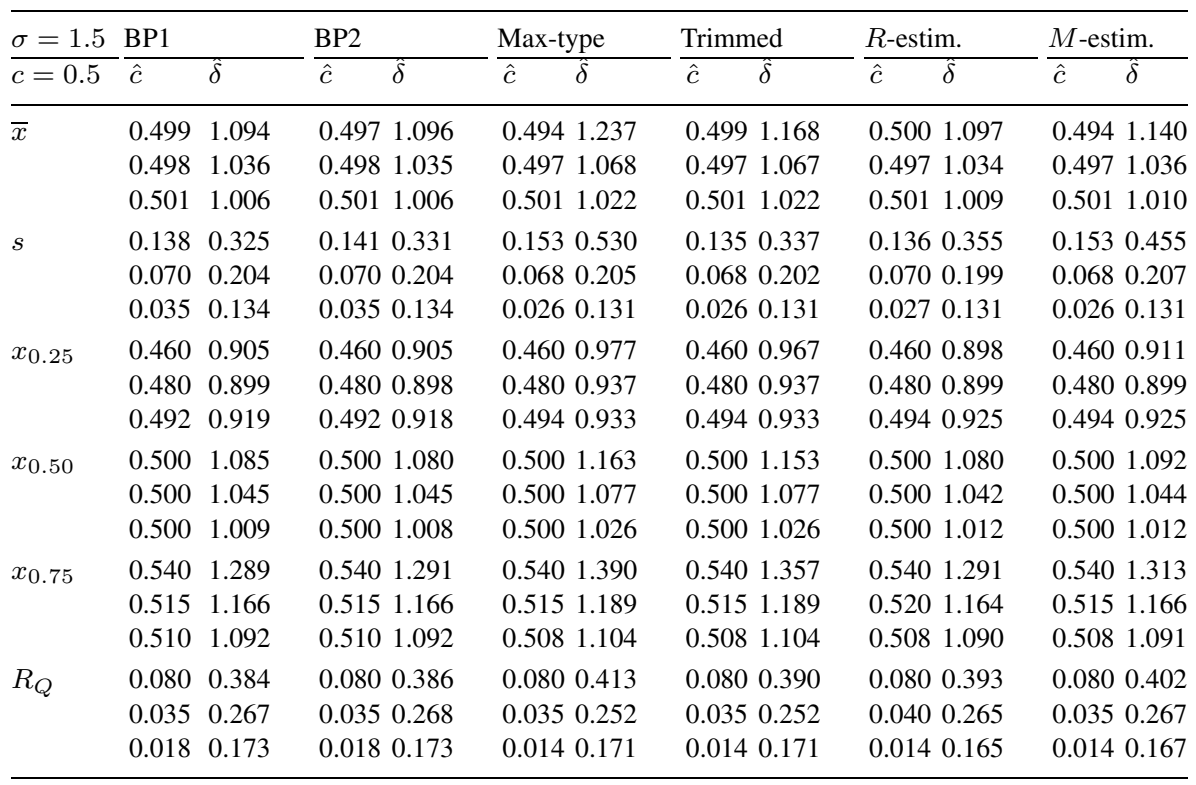

Table 10

Estimates of the parameters $c=0.8$ and $\delta=1$ (simulations $\sigma=1.5, T=100,200,500$ )

\begin{tabular}{|c|c|c|c|c|c|c|c|c|c|c|c|c|}
\hline \multirow{2}{*}{$\begin{array}{l}\sigma=1.5 \\
c=0.8\end{array}$} & \multicolumn{2}{|l|}{ BP1 } & \multicolumn{2}{|l|}{ BP2 } & \multicolumn{2}{|c|}{ Max-type } & \multicolumn{2}{|c|}{ Trimmed } & \multicolumn{2}{|c|}{$R$-estim. } & \multicolumn{2}{|c|}{$M$-estim. } \\
\hline & $\hat{c}$ & $\delta$ & $\hat{c}$ & $\delta$ & $\hat{c}$ & $\hat{\delta}$ & $\hat{c}$ & $\delta$ & $\hat{c}$ & $\delta$ & $\hat{c}$ & $\delta$ \\
\hline \multirow[t]{3}{*}{$\bar{x}$} & 0.722 & 1.047 & 0.720 & 1.046 & 0.723 & 1.278 & 0.726 & 1.176 & 0.717 & 1.052 & 0.723 & 1.119 \\
\hline & 0.779 & 1.033 & 0.780 & 1.034 & 0.775 & 1.097 & 0.778 & 1.093 & 0.773 & 1.030 & 0.775 & 1.038 \\
\hline & 0.796 & 1.007 & 0.797 & 1.009 & 0.798 & 1.036 & 0.797 & 1.033 & 0.798 & 1.010 & 0.798 & 1.013 \\
\hline \multirow[t]{3}{*}{$s$} & 0.197 & 0.507 & 0.201 & 0.515 & 0.224 & 0.802 & 0.200 & 0.560 & 0.218 & 0.575 & 0.224 & 0.614 \\
\hline & 0.110 & 0.276 & 110 & 0.27 & 126 & 0.477 & 0.110 & 0.290 & 0.121 & 0.274 & 126 & 0.372 \\
\hline & 0.041 & 0.186 & 0.041 & 0.185 & 0.034 & 0.188 & 0.033 & 0.181 & 0.033 & 0.182 & 0.034 & 0.186 \\
\hline \multirow[t]{3}{*}{$x_{0.25}$} & 0.710 & 0.808 & 0.710 & 0.811 & 0.720 & 0.962 & 0.720 & 0.938 & 0.710 & 0.848 & 0.720 & 0.854 \\
\hline & 0.780 & 0.872 & & 0.873 & 78 & 0.937 & 80 & 0.928 & 0.775 & 0.870 & 0.778 & 0.880 \\
\hline & 0.792 & 0.881 & 0.793 & 0.881 & 0.792 & 0.906 & 0.792 & 0.904 & 0.792 & 0.884 & 0.792 & 0.885 \\
\hline \multirow[t]{3}{*}{$x_{0.50}$} & 0.790 & 1.050 & 0.790 & 1.050 & 0.800 & 1.252 & 0.790 & 1.193 & 0.790 & 1.073 & 0.800 & 1.121 \\
\hline & 0.800 & 1.037 & 0.800 & 1.035 & 0.800 & 1.100 & 0.800 & 1.097 & 0.800 & 1.034 & 0.800 & 1.038 \\
\hline & 0.800 & 1.018 & 0.800 & 1.019 & 0.800 & 1.040 & 0.800 & 1.040 & 0.800 & 1.014 & 0.800 & 1.019 \\
\hline \multirow[t]{3}{*}{$x_{0.75}$} & 0.820 & 1.331 & 0.820 & 1.328 & 0.830 & 1.557 & 0.820 & 1.448 & 0.820 & 1.331 & 0.830 & 1.383 \\
\hline & 0.820 & 1.21 & 0 & 1.2 & 820 & 1.27 & 0.820 & 1.271 & 0.820 & 1.204 & 0.820 & 1.221 \\
\hline & 0.808 & 1.136 & 0.808 & 1.137 & 0.806 & 1.163 & 0.806 & 1.160 & 0.806 & 1.132 & 0.806 & 1.136 \\
\hline \multirow[t]{3}{*}{$R_{Q}$} & 0.110 & 0.523 & 0.110 & 0.517 & 0.110 & 0.595 & 0.100 & 0.510 & 0.110 & 0.482 & 0.110 & 0.529 \\
\hline & 0.040 & & & & 442 & 0.3 & 0.040 & & 0.045 & 0.334 & 0.042 & 0.342 \\
\hline & 0.016 & 0.255 & 0.015 & 0.256 & 0.014 & 0.257 & 0.014 & 0.256 & 0.014 & 0.247 & 0.014 & 0.250 \\
\hline
\end{tabular}


Table 11

Estimates of the parameters $c=0.2$ and $\delta=1$ (simulations $\sigma=2, T=100,200,500$ )

\begin{tabular}{|c|c|c|c|c|c|c|c|c|c|c|c|c|}
\hline \multirow{2}{*}{$\begin{array}{l}\sigma=2 \\
c=0.2\end{array}$} & \multicolumn{2}{|l|}{ BP1 } & \multicolumn{2}{|l|}{ BP2 } & \multicolumn{2}{|c|}{ Max-type } & \multicolumn{2}{|c|}{ Trimmed } & \multicolumn{2}{|c|}{$R$-estim. } & \multicolumn{2}{|c|}{$M$-estim. } \\
\hline & $\hat{c}$ & $\hat{\delta}$ & $\hat{c}$ & $\hat{\delta}$ & $\hat{c}$ & $\hat{\delta}$ & $\hat{c}$ & $\hat{\delta}$ & $\hat{c}$ & $\hat{\delta}$ & $\hat{c}$ & $\hat{\delta}$ \\
\hline \multirow[t]{3}{*}{$\bar{x}$} & 0.329 & 1.075 & 0.340 & 1.070 & 0.352 & 1.252 & 0.331 & 1.200 & 0.338 & 1.089 & 0.352 & 1.088 \\
\hline & 0.271 & 1.103 & 0.273 & 1.104 & 0.270 & 1.240 & 0.273 & 1.160 & 0.282 & 1.144 & 0.270 & 1.166 \\
\hline & 0.211 & 1.046 & 0.218 & 1.031 & 0.213 & 1.096 & 0.211 & 1.070 & 0.214 & 1.059 & 0.213 & 1.070 \\
\hline \multirow[t]{3}{*}{$s$} & 0.248 & 0.756 & 0.260 & 0.770 & 0.304 & 1.527 & 0.260 & 0.863 & 0.284 & 1.006 & 0.304 & 1.073 \\
\hline & 0.187 & 0.4 & 0.190 & 0.451 & 0.208 & 0.745 & 0.192 & 0.466 & 0.221 & 0.554 & 0.208 & 0.615 \\
\hline & 0.075 & 0.227 & 0.092 & 0.240 & 0.094 & 0.338 & 0.078 & 0.227 & 0.096 & 0.283 & 0.094 & 0.320 \\
\hline \multirow[t]{3}{*}{$x_{0.25}$} & 0.175 & 0.847 & 0.175 & 0.839 & 0.160 & 1.005 & 0.170 & 0.967 & 0.160 & 0.877 & 0.160 & 0.861 \\
\hline & 0.180 & 0.912 & 0.180 & 0.909 & 75 & 0.988 & 0.180 & 0.964 & 175 & 0.935 & 0.175 & 0.931 \\
\hline & 0.188 & 0.893 & 0.186 & 0.875 & 0.187 & 0.915 & 0.188 & 0.913 & 0.188 & 0.894 & 0.187 & 0.895 \\
\hline \multirow[t]{3}{*}{$x_{0.50}$} & 0.220 & 1.154 & 0.220 & 1.154 & 0.215 & 1.389 & 0.220 & 1.322 & 0.220 & 1.199 & 0.215 & 1.225 \\
\hline & 0.205 & 1.112 & 0.205 & 1.104 & 0.200 & 1.191 & 0.205 & 1.169 & 0.205 & 1.149 & 0.200 & 1.136 \\
\hline & 0.200 & 1.056 & 0.200 & 1.034 & 0.200 & 1.081 & 0.200 & 1.074 & 0.200 & 1.066 & 0.200 & 1.062 \\
\hline \multirow[t]{3}{*}{$x_{0.75}$} & 0.420 & 1.485 & 0.450 & 1.495 & 0.510 & 1.839 & 0.425 & 1.689 & 0.445 & 1.573 & 0.510 & 1.631 \\
\hline & 0.273 & 1.3 & 0.282 & 1.33 & 0.265 & 1.460 & 0.275 & 1.380 & 0.282 & 1.384 & 0.265 & 1.383 \\
\hline & 0.214 & 1.208 & 0.220 & 1.199 & 0.214 & 1.238 & 0.214 & 1.229 & 0.215 & 1.218 & 0.214 & 1.217 \\
\hline \multirow[t]{3}{*}{$R_{Q}$} & 0.245 & 0.638 & 0.275 & 0.655 & 0.350 & 0.833 & 0.255 & 0.722 & 0.285 & 0.696 & 0.350 & 0.770 \\
\hline & 0.093 & 0.4 & 02 & & 0.090 & 0.4 & 0.095 & 0.4 & 0.107 & 0.449 & 0.090 & 0.452 \\
\hline & 0.026 & 0.315 & 0.034 & 0.324 & 0.027 & 0.323 & 0.026 & 0.317 & 0.027 & 0.324 & 0.027 & 0.321 \\
\hline
\end{tabular}

Table 12

Estimates of the parameters $c=0.5$ and $\delta=1$ (simulations $\sigma=2, T=100,200,500$ )

\begin{tabular}{|c|c|c|c|c|c|c|c|c|c|c|c|c|}
\hline \multirow{2}{*}{$\begin{array}{l}\sigma=2 \\
c=0.5\end{array}$} & \multicolumn{2}{|l|}{ BP1 } & \multicolumn{2}{|l|}{ BP2 } & \multicolumn{2}{|c|}{ Max-type } & \multicolumn{2}{|c|}{ Trimmed } & \multicolumn{2}{|c|}{$R$-estim. } & \multicolumn{2}{|c|}{$M$-estim. } \\
\hline & $\hat{c}$ & $\hat{\delta}$ & $\hat{c}$ & $\hat{\delta}$ & $\hat{c}$ & $\hat{\delta}$ & $\hat{c}$ & $\hat{\delta}$ & $\hat{c}$ & $\hat{\delta}$ & $\overline{\hat{c}}$ & $\hat{\delta}$ \\
\hline \multirow[t]{3}{*}{$\bar{x}$} & 0.507 & 1.142 & 0.506 & 1.143 & 0.500 & 1.238 & 0.502 & 1.254 & 0.501 & 1.141 & 0.500 & 1.133 \\
\hline & 0.508 & 1.113 & 0.508 & 1.114 & 0.509 & 1.170 & 0.509 & 1.164 & 0.507 & 1.119 & 0.509 & 1.116 \\
\hline & 0.504 & 1.021 & 0.503 & 1.019 & 0.502 & 1.041 & 0.502 & 1.041 & 0.502 & 1.022 & 0.502 & 1.025 \\
\hline \multirow[t]{3}{*}{$s$} & 0.180 & 0.540 & 0.179 & 0.541 & 0.215 & 1.178 & 0.182 & 0.584 & 0.207 & 0.667 & 0.215 & 0.769 \\
\hline & 0.115 & 0.2 & 0.116 & 0.2 & 25 & 0.4 & 14 & 0.258 & 0.121 & 0.293 & 0.125 & 0.367 \\
\hline & 0.063 & 0.166 & 0.064 & 0.166 & 0.056 & 0.165 & 0.056 & 0.165 & 0.052 & 0.162 & 0.056 & 0.165 \\
\hline \multirow[t]{3}{*}{$x_{0.25}$} & 0.430 & 0.907 & 0.430 & 0.907 & 0.420 & 1.022 & 0.430 & 1.004 & 0.415 & 0.951 & 0.420 & 0.923 \\
\hline & 0.465 & 0.951 & 0.465 & $0.94 \mathrm{C}$ & 0.465 & 0.997 & 0.465 & 0.987 & 0.460 & 0.9 & 0.465 & 0.953 \\
\hline & 0.488 & 0.900 & 0.488 & 0.899 & 0.488 & 0.927 & 0.488 & 0.927 & 0.488 & 0.910 & 0.488 & 0.910 \\
\hline \multirow[t]{3}{*}{$x_{0.50}$} & 0.500 & 1.167 & 0.500 & 1.168 & 0.500 & 1.309 & 0.500 & 1.280 & 0.500 & 1.201 & 0.500 & 1.195 \\
\hline & 0.500 & 1.108 & 0.500 & 1.110 & 0.500 & 1.153 & 0.500 & 1.145 & 0.500 & 1.110 & 0.500 & 1.108 \\
\hline & 0.500 & 1.018 & 0.500 & 1.016 & 0.500 & 1.045 & 0.500 & 1.045 & 0.500 & 1.023 & 0.500 & 1.024 \\
\hline \multirow[t]{3}{*}{$x_{0.75}$} & 0.590 & 1.438 & 0.590 & 1.439 & 0.590 & 1.599 & 0.590 & 1.553 & 0.600 & 1.447 & 0.590 & 1.465 \\
\hline & 0.545 & 1.281 & 0.545 & 1.283 & 0.548 & 1.325 & 0.545 & 1.323 & 0.550 & 1.283 & 0.548 & 1.283 \\
\hline & 0.516 & 1.134 & 0.516 & 1.134 & 0.513 & 1.156 & 0.513 & 1.156 & 0.514 & 1.131 & 0.513 & 1.134 \\
\hline \multirow[t]{3}{*}{$R_{Q}$} & 0.160 & 0.531 & & & & & 0.160 & 0.549 & 0.185 & 0.497 & 0.170 & 0.542 \\
\hline & 0.080 & & 0.080 & & 0.083 & & 0.080 & 0.336 & 0.090 & 0.329 & 0.083 & 0.330 \\
\hline & 0.028 & 0.234 & 0.028 & 0.235 & 0.025 & 0.229 & 0.025 & 0.229 & 0.026 & 0.221 & 0.025 & 0.224 \\
\hline
\end{tabular}


Table 13

Estimates of the parameters $c=0.8$ and $\delta=1$ (simulations $\sigma=2, T=100,200,500$ )

\begin{tabular}{|c|c|c|c|c|c|c|c|c|c|c|c|c|}
\hline \multirow{2}{*}{$\begin{array}{l}\sigma=2 \\
c=0.8\end{array}$} & \multicolumn{2}{|l|}{ BP1 } & \multicolumn{2}{|l|}{ BP2 } & \multicolumn{2}{|c|}{ Max-type } & \multicolumn{2}{|c|}{ Trimmed } & \multicolumn{2}{|c|}{$R$-estim. } & \multicolumn{2}{|c|}{$M$-estim. } \\
\hline & $\hat{c}$ & $\hat{\delta}$ & $\overline{\hat{c}}$ & $\hat{\delta}$ & $\overline{\hat{c}}$ & $\hat{\delta}$ & $\overline{\hat{c}}$ & $\hat{\delta}$ & $\overline{\hat{c}}$ & $\hat{\delta}$ & $\overline{\hat{c}}$ & $\hat{\delta}$ \\
\hline \multirow[t]{3}{*}{$\bar{x}$} & 0.671 & 1.013 & 0.665 & 1.020 & 0.655 & 1.271 & 0.669 & 1.185 & 0.648 & 1.016 & 0.655 & 1.096 \\
\hline & 0.727 & 1.085 & 0.725 & 1.088 & 0.725 & 1.234 & 0.726 & 1.171 & 0.718 & 1.113 & 0.725 & 1.114 \\
\hline & 0.789 & 1.035 & 0.789 & 1.038 & 0.790 & 1.080 & 0.795 & 1.078 & 0.792 & 1.043 & 0.790 & 1.060 \\
\hline \multirow[t]{3}{*}{$s$} & 0.240 & 0.748 & 0.246 & 0.751 & 0.290 & 1.436 & 0.251 & 0.850 & 0.291 & 0.982 & 0.290 & 0.931 \\
\hline & 0.187 & 0.445 & 0.190 & 0.450 & 0.208 & 0.791 & 0.191 & 0.472 & 0.212 & 0.506 & 0.208 & 0.565 \\
\hline & 0.085 & 0.235 & 0.085 & 0.238 & 0.094 & 0.443 & 0.070 & 0.232 & 0.071 & 0.232 & 0.094 & 0.267 \\
\hline \multirow[t]{3}{*}{$x_{0.25}$} & 0.565 & 0.785 & 0.550 & 0.7 & 0.530 & 1.0 & 0.580 & 0.9 & 0.530 & 0.8 & 0.530 & 0.851 \\
\hline & 0.710 & 0.876 & 0.710 & 0.880 & 0.712 & 0.980 & 0.710 & 0.949 & 0.710 & 0.902 & 0.712 & 0.913 \\
\hline & 0.788 & 0.884 & 0.788 & 0.886 & 0.788 & 0.931 & 0.788 & 0.928 & 0.786 & 0.897 & 0.788 & 0.900 \\
\hline \multirow[t]{3}{*}{$x_{0.50}$} & 0.770 & 1.091 & 0.770 & 1.102 & 0.780 & 1.383 & 0.780 & 1.289 & 0.775 & 1.173 & 0.780 & 1.191 \\
\hline & 0.795 & 1.098 & 0.795 & 1.100 & 0.795 & 1.211 & 0.795 & 1.188 & 0.795 & 1.121 & 0.795 & 1.126 \\
\hline & 0.800 & 1.045 & 0.800 & 1.045 & 0.800 & 1.088 & 0.800 & 1.085 & 0.800 & 1.046 & 0.800 & 1.058 \\
\hline \multirow[t]{3}{*}{$x_{0.75}$} & 0.830 & 1.479 & 0.830 & 1.479 & 0.840 & 1.825 & 0.830 & 1.702 & 0.830 & 1.532 & 0.840 & 1.583 \\
\hline & 0.820 & 1.310 & 0.820 & & 0.820 & 1.436 & 0.820 & 1.410 & 0.820 & & 0.820 & 1.348 \\
\hline & 0.814 & 1.179 & 0.814 & 1.179 & 0.812 & 1.214 & 0.812 & 1.209 & 0.812 & 1.180 & 0.812 & 1.183 \\
\hline \multirow[t]{3}{*}{$R_{Q}$} & 0.265 & 0.694 & 0.280 & 0.691 & 0.310 & 0.791 & 0.250 & 0.769 & 0.300 & 0.704 & 0.310 & 0.732 \\
\hline & 0.110 & 0.434 & 0.110 & 0.434 & 0.108 & 0.456 & 0.110 & 0.461 & 0.110 & 0.443 & 0.108 & 0.436 \\
\hline & 0.026 & 0.296 & 0.026 & 0.294 & 0.024 & 0.283 & 0.024 & 0.281 & 0.026 & 0.283 & 0.024 & 0.283 \\
\hline
\end{tabular}

\section{Conclusions}

Simulation study indicates that all mentioned methods of change point detection (statistical methods and method based on basis pursuit algorithm with Heaviside dictionary) give us similar results of change point detection (the parameter $c$ ) for small or large values of the standard deviation of the error term. BP method seems to yield a slightly better estimate of the parameter $\delta$ for large values of standard deviation (see Tables 2-13). Precision of estimates goes down with decreasing sample length for all mentioned kind of estimates (the standard deviation $s$ and the interquartile range are larger for $T=100$ than for $T=500$ ), which is expectable. Simulation study confirms another assumed result that with the increasing variance of additive white noise in the model the accuracy of estimates debases. BP1 approach using ideal centering provides better estimates than BP2 in which the centering parameter is estimated from the process. However, the difference diminishes for large sample length. We studied the change points in three locations, in the middle of the process $c=0.5$ and at points $c=0.2$ and 0.8 . According to the simulations we can say that the standard deviation of estimates of the change point in smaller for $c=0.5$ than in the case that the change point is not in the middle of the process, but the interquartile range is approximately the same in all locations. On the other hand, the estimates of the parameter $\delta$ have the larger standard deviation and also the interquartile range whenever the change point is not situated in the middle of the process.

The basis pursuit approach proposes an alternative technique of the change point detection. This paper deals with the introductory empirical comparative study for the simplest case of detecting one change point. The outlined method can be used for detection 


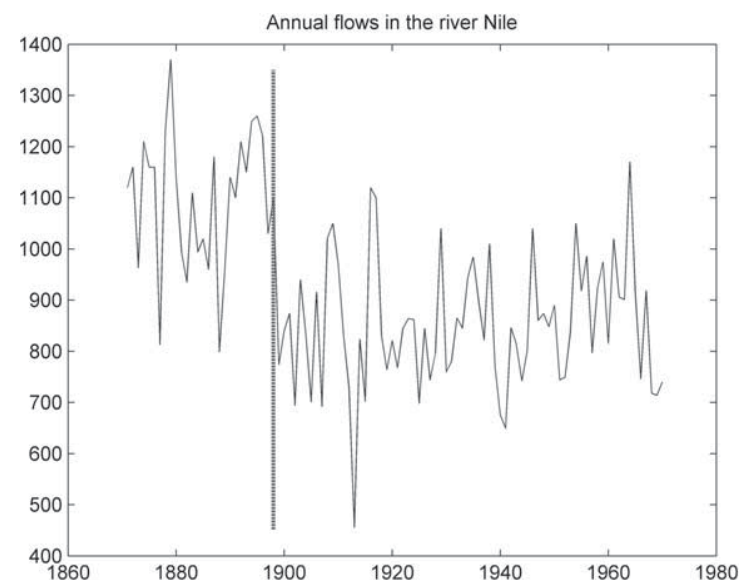

Fig. 5. The annual flows (in billions of the cubic meters) in the river Nile in Aswan (Egypt) during the years 1871-1970.

of two or more change points - the authors have already started to work on this problem or another sort of change point with a dictionary $G$ of different kind.

The change point detection techniques may be useful for instance in modeling economical or environmental time series where jumps can occur.

Acknowledgement. This research was supported by the grants GAČR P402/10/P209 and MSM0021622418.

\section{References}

Antoch, J., Hušková, M., Jarušková, D. (2000). Change point detection. In: 5th ERS IASC Summer School, IASC 2000.

Bruckstein, A.M., Donoho, D.L., Elad, M. (2009). From sparse solutions of systems of equations to sparse modeling of signals and images. SIAM Review, 51(1), 34-81.

Csörgö, M., Horváth, L. (1997). Limit Theorems in Change-Point Problem. Wiley, New York.

Chen, S.S., Donoho, D.L., Saunders, M.A. (1998). Atomic decomposition by basis pursuit. SIAM Journal on Scientific Computing, 20(1), 33-61 (2001 reprinted in SIAM Review, 43(1), 129-159).

Christensen, O. (2003). An Introduction to Frames and Riesz Bases. Birkhäuser.

Janušauskas, A., Marozas, V., Lukoševičius, A., Sörnmo, L. (2010). Detection of hearing loss in audiological frequencies from transient evoked otoacoustic emissions. Informatica, 21(2), 191-204. http: / / www.mii.1t/Informatica/pdf/INF0785.pdf.

Saunders, M.A. (1997-2001). pdsco.m: MATLAB code for minimizing convex separable objective functions subject to $A x=b, x \leqslant 0$.

Veselý, V. (2001-2008). framebox: MATLAB toolbox for overcomplete modeling and sparse parameter estimation.

Veselý, V., Tonner, J., Hrdličková, Z., Michálek, J., Kolář, M. (2009). Analysis of PM10 air pollution in Brno based on generalized linear model with strongly rank-deficient design matrix. Environmetrics, 20(6), 676-698. http: / / dx.doi.org/10.1002/env. 971.

Veselý, V., Tonner, J. (2006). Sparse parameter estimation in overcomplete time series models. Austrian Journal of Statistics, 35(2-3), 371-378.

http: / / www. stat.tugraz.at/AJS/ausg062+3/062Vesely.pdf. 
Zelinka, J., Veselý, V., Horová, I. (2004). Comparative study of two kernel smoothing techniques. In: Proceedings of the Summer School DATASTAT'2003, Svratka, Horová, I Mathematica 15, Masaryk University, Brno, Czech Rep., pp. 419-436. http: / / www. econ.muni.cz/ vesely/papers / datastat03.pdf.

J. Neubauer graduated from Masaryk University, Czech Republic, in 1998. He received the doctoral degree in applied mathematics, University of Ostrava, Czech Republic, in 2006. He is head of Processes Modeling Group at the Department of Econometrics, University of Defence, Czech Republic. His research interests cover time series analysis and change point detection. He is a member of The International Association for Statistical Computing (IASC).

V. Veselý graduated in mathematics (MSc) from Masaryk University, Brno, Czech Republic, in 1972. From the same university he received the doctoral RNDr and $\mathrm{PhD}(\mathrm{CSc})$ degrees in algebra and number theory in 1974 and 1990, respectively. In 1982-1991 he was in positions of researcher and later on research scientist at the Institute of Physical Metallurgy of the Czechoslovak Academy of Sciences in Brno (currently the Institute of Physics of Materials of the Czech Academy of Sciences) working with the Electrical and Magnetic Properties Group in the field of FFT-based methods for Mössbauer spectroscopy. Since 1994 he holds the full-time and part-time associate professor positions at the Masaryk University and Brno University of Technology, respectively. His current professional interests include theory and algorithms for data processing: time series analysis, filtration/smoothing, harmonic and nonharmonic spectral decomposition (Fourier, wavelet and other related transforms, frames) mainly focussing on applied sparse modeling. He is a member of the Union of Czech Mathematicians and Physicists, AMS and SIAM.

\title{
Pasikeitimo momento nustatymas taikant išretintą parametru vertinima
}

\author{
Jiri NEUBAUER, Vitezslav VESELY
}

Straipsnyje nagrinėjamas vienmačiu procesu pasikeitimo momento nustatymas pritaikant išretintą parametrų vertinimą pertekliniame modelyje. Stochastinis procesas su vidurkio pasikeitimu yra ịvertinamas pasinaudojus Hevisaido funkciju rinkiniu. Bazinis tikslinis algoritmas yra naudojamas išretintu parametrų ịverčiams gauti. Nagrinejjamas stochastinio proceso pasikeitimo momento nustatymo metodas yra palyginamas kompiuterinio modeliavimo būdu su keliais standartiniais statistiniais metodais. 